\title{
Research on Distributed Harmony-SE Model Integration Method for Complex UAV System
}

\author{
Guang Zhan, Miao Wang, Zhixiao Sun, Yuanjie Lu, and Yang Bai
}

\begin{abstract}
The distributed modeling of complex unmanned aerial vehicle (UAV) system is faced with the problem of multiple use cases and multiple models integration. After studying the purpose and principles of model integration, we proposed a Harmony-SE based tight coupling integration method for the white box functional logic model of UAV system, model integration process was described in detail. The whole system model integration of complex UAV and the whole system functional logic verification based on state machine diagram are realized, which is of great value to realize the collaborative design and parallel modeling of multiple use cases of complex UAV system.
\end{abstract}

